\title{
Traitement des gaz
}

\author{
- Gas Treatment -
}

par Xavier Richelmy

COS Ingénierie

This paper illustrates through four particle (dust or drop) carrying over cases, the need of theoretical research on fundamental mechanisms of the particle formation, their growth, the hydrodynamics and their catchment, with the idea that energy should be saved.

\section{INTRODUCTION}

Les entraînements diphasiques dans les effluents gazeux sont un domaine où des technologies émergentes ont leur place, et où, il faut bien l'admettre, les mécanismes de transfert de masse sont mal connus.

Nombreux sont les secteurs industriels concernés par les problèmes d'entraînements vésiculaires dans les gaz : la chimie, la chimie fine, la métallurgie, etc.

Pour respecter les nouvelles (et les futures) directives françaises et européennes sur la protection de l'environnement et à cause également de leur impact sur la santé humaine, les vésicules de gaz nocifs sont désormais sur la sellette de l'administration.

Il s'agit de gaz acides (brouillard sulfurique) ou basiques (ammoniac). La captation des composés organiques volatils (C.O.V.) correspond en partie à la même problématique.

Quant aux problèmes de dépoussiérage, surtout sur des granulométries très fines ou très étalées, des techniques de captation existent, mais leur optimisation énergétique n'est certainement pas définitive aujourd'hui.

L'objectif de cet exposé n'est pas de lister les technologies disponibles sur le marché, ni de présenter les secteurs industriels concernés par ces problèmes.

Nous souhaitons simplement illustrer sur 4 types d'entraînement particulaire (poussières ou gouttelettes) le besoin d'une recherche théorique des mécanismes fondamentaux de la formation de ces particules, de leur croissance, de l'hydrodynamique et de leur captation dans un souci d'optimiser les dépenses énergétiques.

\section{II — PROBLÈMES DE DÉPOUSSIÉRAGE}

Commençons cette brève description des problèmes rencontrés par l'industrie aujourd'hui, au niveau de la limitation des rejets gazeux, par un aspect a priori connu : l'arrêt des poussières.

On appelle en ce sens "poussière" toute particule, ou amas de particules minérales, qui peuvent être entraînés par un flux gazeux (air, fumées,...). Ce sont des métaux lourds, des oxydes métalliques, des cristaux, etc. dont la dispersion granulométrique peut couvrir un spectre très large : de moins de 1 micron à plus de $1 \mathrm{~mm}$.

L'approche "classique" du dépoussiérage consiste à filtrer directement, ou après agglomération (et neutralisation) éventuelle, ces particules plus ou moins fines, à l'aide de filtresmanches, d'électrofiltres, selon des modes de fonctionnement totalement secs ou par voie humide.

En règle générale, plus la particule est fine et plus il faut d'énergie pour la capter.

En fait, comme le problème des poussières est rarement isolé, la solution n'est jamais optimisée pour le seul traitement de ces poussières. Par exemple, pour capter les fines contenues dans les fumées issues d'un incinérateur de déchets industriels spéciaux, il faut prendre en compte l'acidité des gaz, leur température, les problèmes de corrosion, la présence de molécules hologénées, et beaucoup d'autres paramètres.

C'est pourquoi, à ce jour, la connaissance des mécanismes du dépoussiérage fin, dans ses applications pratiques, est peu développée chez les industriels du secteur, qui privilégient les solutions technologiques aux problèmes posés (matériaux 
adaptés, raclage ou décolmatage en ligne, batteries de séparateurs en série).

La mauvaise maîtrise des phénomènes en jeu conduit parfois à des situations où une filtration trop poussée nuit à l'efficacité globale d'un traitement.

\section{III — PROBLÈMES DE VÉSICULES SULFURIQUES}

Il est très difficile d'arrêter un brouillard sulfurique (molécules de $\mathrm{SO}_{3}$ gazeux). Dans la Littérature et même au niveau des industriels spécialistes du domaine, il est également très difficile de trouver une distribution de la taille des gouttelettes formant le brouillard.

Les abaques ou résultats d'expériences correspondent à des gaz secs en général (sans eau) et à des températures précises.

Peu de spécialistes sont capables d'expliquer la formation des gouttes, et cette lacune au plan théorique chez la plupart des ingénieurs "de terrain" va jusqu'à l'impossibilité de calculer de façon sûre, les pertes de charge dans une gaine où circule un air chaud, saturé en eau, et contenant du brouillard sulfurique.

Les secteurs industriels concernés sont limités, mais l'enjeu est important puisqu'il s'agit de la revalorisation des effluents liquides sulfuriques.

\section{IV — PROBLÈMES DE VÉSICULES D'AMMONIAC}

Pour illustrer le manque de données accessibles par les Ingénieurs "de terrain" sur la formation des vésicules, nous allons citer le cas d'un stockage d'ammoniac.

Nous savons que l'emploi de l'ammoniac comme fluide frigorigène de substitution aux $\mathrm{CFC}$, est de plus en plus répandu en milieu industriel où le besoin en froid se fait à des niveaux de température négatifs. Cela est également valable pour des applications de climatisation de confort, ou des patinoires, etc.

Or, les installations frigorifiques correspondant aux besoins d'un abattoir par exemple, contiennent un volume d'ammoniac supérieur au seuil pour lequel la Législation prévoit un équipement de neutralisation.

En effet, en cas de rupture d'une canalisation dans laquelle circulait l'ammoniac liquide, celui-ci se déverse au sol avant de s'évaporer partiellement, par l'effet de la détente thermodynamique.

$\mathrm{Si}$ la vaporisation était totale sous forme de gaz, le calcul serait facile. Or une partie de l'ammoniac s'évapore sous forme de vésicules, selon des mécanismes et une hydrodynamique plutôt empiriques.

Le dimensionnement des équipements de sécurité (tour de neutralisation, par exemple) repose sur des hypothèses de débit et de concentration, qui ne sont donc pas très précises, et l'optimisation technique et financière de ces matériels désormais obligatoires, est loin d'être évidente. L'enjeu dans ce cas, est lourd au niveau économique, pour des collectivités ou des industriels à qui la réglementation impose ces investissements en cas d'hypothétiques accidents.

\section{V — PROBLÈMES DE COMPOSÉS ORGANIQUES VOLATILS}

Dans le secteur des métiers de l'Environnement, les Composés Organiques Volatils (C.O.V.) sont désormais ceux qui posent le plus de problèmes aux industriels : solvants de rinçage, ou solvants nécessaires pour les applications d'enduction, d'encollage, de revêtement, etc.

La prochaine promulgation en Droit Français de la Directive européenne les concernant va rendre cruciale la mise à disposition de technologies acceptables économiquement et efficaces techniquement, sachant que les valeurs admises pour les rejets sont parfois très basses (jusqu'à $20 \mathrm{mg} / \mathrm{Nm}^{3}$ ).

A côté des solutions de destruction par oxydation thermique, qui sont les plus répandues à ce jour, il existe des solutions de récupération des solvants qui permettent une notion de temps de retour sur investissement.

L'adsorption sur charbon actif ou autre support est également bien maîtrisée quand elle est possible.

Mais, pour des raisons de compatibilité "culturelle" (la manipulation de charbon en pharmacie n'est pas usuelle), ou chimique (présence de molécules type cétones ou nitriles) dans les gaz, les technologies d'adsorption ne conviennent pas toujours.

Nous constatons en revanche que si "culturellement" la cryocondensation (refroidissement des gaz par échangeur avec de l'azote liquide), est une technologie tout à fait compatible avec les usages de la chimie fine et de la pharmacie, le mécanisme de la condensation de vapeurs de solvants semble mal connu. En effet, la plupart des références actuelles fonctionnent plutôt difficilement.

Une autre technique, par absorption dans une huile adaptée, s'avère elle aussi insuffisante dès lors qu'il y a présence de vésicules (cas des plastifiants par exemple).

\section{VI $\square$ CONCLUSION}

Le but de cet exposé est de mettre en évidence le besoin d'une recherche théorique, à l'heure actuelle pour mettre à disposition des ingénieurs et techniciens les outils nécessaires à une bonne détermination des paramètres de fonctionnement, pour rédiger les cahiers des charges ou dimensionner les appareils.

Un vaste champ d'application concerne les nouveaux secteurs de l'Environnement, notamment les problèmes de captation des vapeurs de solvants, de plastifiants en chimie fine, pharmacie et parachimie.

Mais même des techniques "éprouvées" sont susceptibles d'amélioration pour que les cas d'application extrêmes ne réservent pas des surprises désagréables et coûteuses. 